\title{
A Case of Streptococcus Suis Meningitis with Visual Impairment And Hearing Loss Diagnosed by Metagenomic Next-Generation Sequencing
}

\author{
Haina Zhao', Lanlan Chen', Lin Zhu', Tingting Qiao, Peipei Liu, Cancan Ma, Yao Xu* and Li Jiang*
}

Department of Neurology, Northern Jiangsu People's Hospital, Medical College of Yangzhou University, China

"Corresponding author: Yao Xu, Department of Neurology, Northern Jiangsu People's Hospital, Medical College of Yangzhou University, Yangzhou, 225001, China

\section{Co-corresponding authors}

Li Jiang, Department of Neurology, Northern Jiangsu People's Hospital, Medical College of Yangzhou University, Yangzhou, 225001, China

\begin{abstract}
Background: Streptococcus suis is responsible for several kinds of zoonosis worldwide. It can cause meningitis, sepsis, osteoarthritis, endocarditis, endophthalmitis, and other diseases. Human infection typically occurs in occupational settings associated with pig husbandry or pork processing. At present, Metagenomic Next-Generation Sequencing (mNGS) is prominent testing method for achieving an early and rapid diagnosis of some infectious diseases.

Case Presentation: A 48-year-old Chinese man who denied exposure to pigs or pork was to admitted the hospital because he had experienced a fever for three days, visual impairment, and hearing loss for one day. After a series of examinations and laboratory tests, Streptococcus suis was detected in the aqueous humor culture as well as the vitreous humor and cerebrospinal fluid with mNGS. But bacteria were not detected in the blood or cerebrospinal fluid culture. The patient's condition improved, and he was discharged after 2 weeks of active treatment. However, at present, poor vision in the right eye, poor hearing in the right ear and recurrent vertigo remain.
\end{abstract}

Conclusions: In a patient presenting with meningitis, vision loss, and/or hearing loss, Streptococcus suis infection should be strongly suspected regardless of the patient's occupation. mNGS has excellent diagnostic value to determine which was the etiological agent for infectious diseases.

Keywords: Streptococcus suis; meningitis; visual impairment; hearing loss; metagenomic next-generation sequencing.

Abbreviations: mNGS: Metagenomic Next-Generation Sequencing; S. suis: Streptococcus suis; CT: Computed Tomography; MRI: Magnetic Resonance Imaging; CSF: Cerebrospinal Fluid; WBCs: White Blood Cells

\section{Background}

Streptococcus suis (S. suis) is a common zoonotic pathogen [1]. S. suis infection can result in purulent meningitis, sepsis, osteoarthritis, endocarditis, endophthalmitis, and other diseases [2]. Given its varied clinical symptoms and lack of specificity, S. suis infection is easily missed or misdiagnosed. If the patient is not diagnosed and treated in a timely manner, disability and even death can occur. In 1968, Denmark was the first to report a human infection with S. suis [3] followed by the United States, France, Germany, Thailand, Japan, China, and other countries. We found that Asia, especially Thailand, Vietnam, and China, has a higher prevalence of S. suis infection than other areas of the world. Two public health events involving human infection with S. suis occurred in China in 1998 and 2005 [4], and sporadic cases have been reported since then in many parts of the world. There have been many reports of meningitis with hearing loss [5] and fewer reports of meningi- tis with vision loss or arthritis [6], but meningitis accompanied by both visual impairment and hearing loss is rare. Recently, metagenomic next-generation sequencing (mNGS) has been applied to pathogen detection to achieve early and rapid diagnosis [7]. When there is clinical suspicion of an infectious disease after an anti-infection treatment, mNGS has an advantage over conventional detection methods. Here, we report a case of $\mathrm{S}$. suis meningitis with visual impairment and hearing loss diagnosed by mNGS.

\section{Case Presentation}

In June 2020, a 48-year-old Chinese man with a history of hypertensive blood pressure was admitted to the local clinic. He was a site manager who denied eating contaminated food, catching cold, or skin damage recently. His symptoms included a high fever for 3 days, vomiting, chills, fatigue. He was treated by anti-infection therapy with intravenous penicillin (the spe- 
cific treatment dose is unknown), but the disease progressed quickly, decreased vision occurred in his right eye complicated by bilateral hearing loss. The patient was transferred to our hospital for further treatment.

At admission, his body temperature was $37{ }^{\circ} \mathrm{C}$, his pulse rate was 75 beats per minute, his blood pressure was 120/81 $\mathrm{mmHg}$, and his respiratory rate was 20 breaths per min. An ophthalmic physical examination showed that the visual acuity of the right eye was only light perception, the visual acuity of the left eye was 0.4 , and the conjunctiva of the right eye was congested with pus in the anterior chamber. No abnormalities were apparent upon neurological examination. The laboratory results showed no notable values except for an elevated hypersensitive C-reactive protein level $(40.68 \mathrm{mg} / \mathrm{L}$; normal range, 0-10 mg/L), which was associated with a faster erythrocyte sedimentation rate $(37 \mathrm{~mm} / \mathrm{hr}$; normal range, $0-15 \mathrm{~mm} / \mathrm{hr}$ ). No abnormalities were found on orbital Computed Tomography $(\mathrm{CT})$, a plain cranial Magnetic Resonance Imaging (MRI) scan, or an enhanced cranial MRI scan. The results of audiometric testing showed bilateral sensorineural hearing loss that was severe in the right ear. The patient was diagnosed with severe endophthalmitis with bilateral deafness. Meningitis were not observed at that time.

The patient received an intravitreal injection of vancomycin in the right eye from ophthalmologists after a blood sample and pus in the aqueous humor were obtained for bacterial culture and a sample of the vitreous humor was taken for mNGS (Beijing Giantmed Medical Diagnostics Laboratory). The patient was treated with empirical intravenous clarithromycin and piperacillin-tazobactam to cover sensitive bacteria. On the second day of admission, the patient's right eye underwent standard vitrectomy, phacoemulsification and oil tamponade via a posterior approach. At $24 \mathrm{~h}$ after surgery and medication, the hearing in the left ear was miraculously improving, and the patient was not experiencing fever. Four days after admission, bacteria were not detected in the blood culture. However, S. suis was detected in the aqueous humor culture, and $\mathrm{S}$. suis was detected in the vitreous humor with mNGS. The identified number of unique reads mapped to the $\mathrm{S}$. suis genome sequence was 4,921 with high abundance values. On that day, the patient received a lumbar puncture because $\mathrm{S}$. suis is the most common cause of meningitis[2]. The Cerebrospinal Fluid (CSF) was clear with a pressure of $280 \mathrm{mmH} 2 \mathrm{O}$ and contained $55 \times 10^{\wedge} 6 / \mathrm{L}$ White Blood Cells (WBCs) (reference range, $\left.<8 \times 10^{\wedge} 6 / \mathrm{L}\right)$, mainly mononuclear cells $(96.3 \%)$. CSF protein was $1.48 \mathrm{~g} / \mathrm{L}$ (reference range, $0.2-0.4 \mathrm{~g} / \mathrm{L}$ ), and chloride and glucose were normal, the immediate blood glucose was nor$\operatorname{mal}(4.5 \mathrm{mmol} / \mathrm{L})$. The CSF culture was negative. A CSF sample was immediately sent for pathogen detection by NGS at the Vision Medical Research Institute. The sample was sequenced on the Illumina NextSeq500 platform using a 75-cycle, single-end sequencing run, and the mNGS of the CSF again detected S. suis. However, the number of identified unique reads mapped to the S. suis genome sequence was only 80 with genome coverage of $0.48 \%$ (Figure 1), likely due to the prior treatments. After confirming S. suis infection, the treatment plan was adjusted. Clarithromycin and piperacillin-tazobactam were stopped immediately. Then, $2 \mathrm{~g}$ of ceftriaxone, which is sensitive to S. suis, and $125 \mathrm{~mL}$ of $20 \%$ mannitol were injected intravenously every $12 \mathrm{~h}$. Neurotrophic drugs and low-dose prednisolone were orally administered to improve his hearing. The condition of the patient improved. The purulent discharge from the anterior chamber of the right eye disappeared gradually, and the wound healed well. However, the vision in the right eye and hearing in the right ear remained poor. On the 12th day after admission, a repeat lumbar puncture revealed decreased pressure $(215 \mathrm{mmH} 2 \mathrm{O})$, decreased white blood cell numbers $(21 \times 106 / \mathrm{L})$, and reduced protein levels $(0.75 \mathrm{~g} / \mathrm{L})$. Thus, he was discharged from the hospital after 2 weeks. Two months later, at a follow-up in the outpatient clinic, he still complained of poor vision in the right eye and poor hearing in the right ear with recurrent vertigo.

\section{Discussion and Conclusions}

S. suis is Gram-positive bacterium with a strong pathogenic effect that commonly persists in the mucous membrane, nasal cavity, and gastrointestinal tract of pigs. It may infect humans through damaged wounds, mucous membranes, the digestive tract, and other pathways [4]. Patients often engage in close contact with pigs or eat infected or raw pork. However, according to the literature, less than half of S. suis patients in Asian countries reported exposure to pigs or pork [8]. In our case, the patient had not engaged in close contact with pigs and denied eating raw or infected pork. Moreover, other people who ate with the patient had no similar symptoms. However, S. suis is not an opportunistic pathogen despite its strong pathogenicity. From this, we hypothesize that the patient had an inconspicuous wound that may have been the pathway for infection before the onset of the disease.

S. suis infection can cause purulent meningitis, sepsis, osteo-

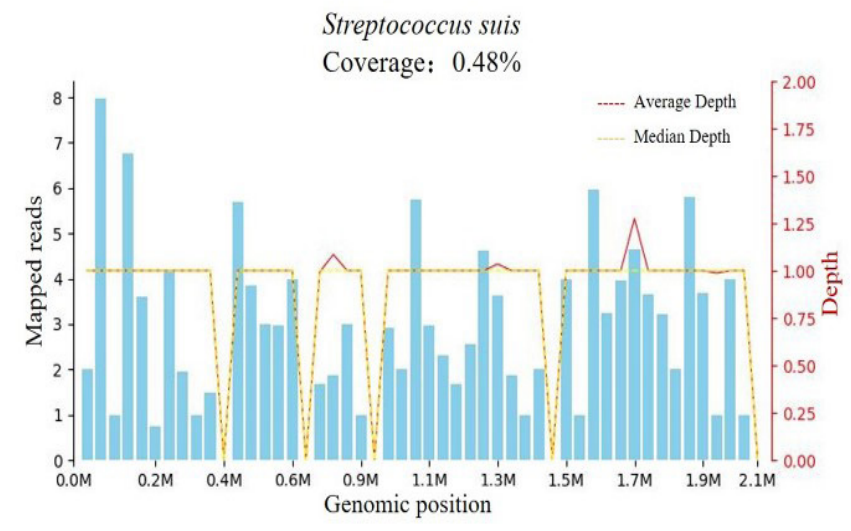

Distribution of Streptococcus suis （Bacteria $\mathrm{N}=925$ reads)

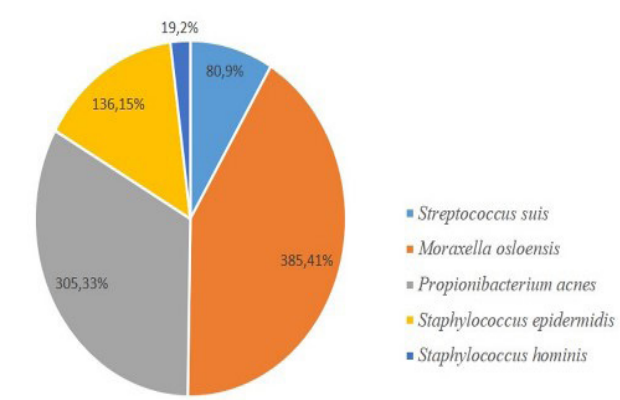

Fig.1 Diagnosis of S. suis infection using mNGS. (A) The majority of reads mapped to the S. suis genome, with coverage of $0.48 \%$. (B) A total of 925 sequence reads corresponded to S. suis, accounting for $8.65 \%$ of microbial reads. 
arthritis, endocarditis, endophthalmitis, endophthalmitis, and other diseases. Meningitis is the most common clinical manifestation [2]. Common pathogenic microorganisms may lead to local tissue injury, whereas S. suis infection causes extensive tissue disruption in multiple tissues or organs. The incidence of sequelae, particularly hearing loss, in patients with S. suis infection is significantly greater than that in patients with other bacterial infections. One study from Vietnam showed that $66.4 \%$ of patients with $\mathrm{S}$. suis meningitis developed hearing loss during hospital admission [9]. This finding was also confirmed in a meta-analysis based on 24 studies with a total of 913 patients, in which the rate of mortality due to S. suis meningitis was low, but hearing loss was common [2]. An animal study showed that due to the dissemination of the exotoxin, $\mathrm{S}$. suis in the blood is believed to enter the perilymph via the cochlear aqueduct, causing suppurative inflammation [10]. This eventually lead to hearing loss in the cochlea. However, this theory has not been confirmed in humans to date. A previous study [11] stated that some genes within S. suis promote adhesion to the ocular epithelium. This may explain why S. suis infections are more likely to cause endophthalmitis than other bacterial infections, such as pneumococcal, Listeria, tuberculosis, and cryptococcal infections. It was thereby inferred that S. suis infection should be highly suspected if the patient suffers from meningitis with impaired vision and/or hearing.

The diagnosis of $\mathrm{S}$. suis meningitis is based on clinical signs and typical CSF findings. Isolation of S. suis from a sterile site (such as the blood or CSF) or the lesion site results in a definitive diagnosis [4]. However, in a previous report on S. suis meningitis, $66.7 \%$ of patients had $\mathrm{S}$. suis isolated from the CSF, $50 \%$ had S. suis isolated from the blood, and $25 \%$ had S. suis isolated from both fluids [12]. It is more difficult to detect S. suis in patients with antibiotic use before treatment. In this case, the symptoms of visual impairment and hearing loss were prominent in the early stage of the disease, but the meningitis symptoms were easy to ignore. Lumbar puncture was performed after realizing that $\mathrm{S}$. suis infection was a possibility. However, the blood culture, CSF culture, and properties of the CSF of the patient were not sufficient to support a diagnosis of S. suis infection. S. suis meningitis was eventually definitively diagnosed in the patient based on the results of mNGS. mNGS is a high-throughput approach that can directly detect the genetic material of pathogens in clinical samples, yielding almost complete coverage of the pathogens causing infections, including those that could not be cultured [7]. This technique can support the early and rapid diagnosis of infectious diseases even if use antibiotics before testing. There is no doubt that the mNGS approach was the key to rapidly detect the cause of infection, additionally it could have revealed potential mutation to antibitic target genes, associated with resistance, with no need of isolation and culture of the organism for conventional susceptibility tests. Although there have been few reports of infections diagnosed based on mNGS, the future of mNGS is promising. Using mNGS can facilitate the achievement of an early and accurate diagnosis and avoid misdiagnoses and missed diagnoses, thereby improving outcomes.

The principles for treating S. suis meningitis include mainly etiological therapy, similar to those for treating most other types of bacterial meningitis [9]. The early use of sensitive antibiotics is vital. Ceftriaxone is the antibiotic that is most commonly used clinically through the intravenous administration of $2 \mathrm{~g}$ ceftriaxone every $12 \mathrm{~h}$ for at least 10 days. If the treatment effect is unsatisfactory after 1 week, the antibiotics should be adjusted in a timely manner. The literature has pointed out that a long course of treatment should be required for recrudescent S. suis meningitis [13]. In addition, a previous report showed that approximately $66.4 \%$ of patients with S. suis meningitis suffered from hearing loss during hospitalization; the hearing loss remained in $44.7 \%$ of patients after discharge, and the early use of hormones was sufficient to reduce hearing loss [14]. In our case, the patient spent only three days from onset to vision and hearing loss. With effective anti-infection treatment and appropriate hormone treatment, his vision and hearing loss were slightly improved. However, two months after discharge, the patient still experienced severe hearing loss. This result is consistent with those in previous studies.

S. suis infection is uncommon in clinical settings, and its diversified symptoms and low specificity make clinical diagnosis difficult. Patients may present with meningitis, vision loss, and/or hearing loss. S. suis infection should be highly suspected regardless of the patient's occupational background. As a convenient and promising diagnostic method, mNGS has excellent diagnostic value to determine which was the etiological agent for infectious diseases. It is hoped that this case report can improve clinicians' knowledge of S. suis infection and reduce missed diagnoses and misdiagnoses.

\section{Declarations}

\section{Ethics approval and consent to participate}

Ethical approval for this study was granted by the ethics committee of the Northern Jiangsu People's Hospital.

\section{Consent for publication}

Written informed consent was obtained from the patient for publication of this case report and any accompanying images. A copy of the written consent for publication from the patient is available for review by the Editor-in-Chief of this journal.

\section{Availability of data and materials}

All data generated or analysed during this study are included in this published article.

\section{Competing interests}

The authors declare that they have no competing interests.

\section{Funding}

Not applicable.

\section{Authors' contributions}

Yao $\mathrm{Xu}$ is the principal investigator who drafted the original manuscript. Haina Zhao participated in laboratory analysis of CSF and drafted parts of the original manuscript. Lanlan Chen and Lin Zhu were involved in case and sample collection as well as analysis or interpretation of data. Li Jiang revised the manuscript. Tingting Qiao was involved in case and sample collection and analysis of the data. Peipei Liu and Cancan Ma analyzed and interpreted the data. All authors have read and approved the manuscript.

\section{Acknowledgements}

The authors are very grateful to the patient for participating in this study. 


\section{References}

1. Gottschalk M, Higgins R, Jacques M, Mittal KR, Henrichsen J. Description of 14 new capsular types of , J Clin Microbiol 1989; 27(12): 2633-2636.

2. van Samkar A, Brouwer MC, Schultsz C, van der Ende A, van de Beek D. Meningitis: A Systematic Review and Meta-analysis, PLoS Negl Trop Dis 2015; 9(10): e0004191.

3. Arends JP, Zanen HC. Meningitis caused by Streptococcus suis in humans, Rev Infect Dis 10(1) (1988) 131-137.

4. Lun ZR, Wang QP, Chen XG, Li AX, Zhu XQ. Streptococcus suis: an emerging zoonotic pathogen, Lancet Infect Dis 2007; 7(3): 201-209.

5. Salaneuve K, Meunier A, Aubry K. Bilateral total deafness after preparation of wild boar meat, Eur Ann Otorhinolaryngol Head Neck Dis 2020; 137(5): 419-421.

6. Rayanakorn A, Katip W, Lee LH, Oberdorfer P, Endophthalmitis with bilateral deafness from disseminated infection, BMJ Case Rep 2019; 12(2).

7. Gu W, Deng X, Lee M, Sucu YD, Arevalo S, Stryke D, et al. Rapid pathogen detection by metagenomic next-generation sequencing of infected body fluids, Nat Med, 2020.

8. Ma E, Chung PH, So T, WongL, Choi KM, Cheung DT, et al.
Collaborative Study Group on infection in Hong, K, Streptococcus suis infection in Hong Kong: an emerging infectious disease?, Epidemiol Infect, 2008; 136(12): 1691-1697.

9. Hlebowicz M, Jakubowski P, Smiatacz T. Streptococcus suis Meningitis: Epidemiology, Clinical Presentation and Treatment, Vector Borne Zoonotic Dis, 2019; 19(8): 557-562.

10. Dom"anguez-Punaro MC, Koedel U, Hoegen T, Demel C, Klein M, Gottschalk M. Severe cochlear inflammation and vestibular syndrome in an experimental model of infection in mice, Eur J Clin Microbiol Infect Dis 2012; 31(9): 2391-2400.

11. Antic I, Brothers KM, Stolzer M, Lai H, Powell E, Eutsey R, et al. Hiller, Gene Acquisition by a Distinct Phyletic Group within Streptococcus pneumoniae Promotes Adhesion to the Ocular Epithelium, mSphere 2017; 2(5).

12. Suankratay $C$, Intalapaporn $P$, Nunthapisud $P$, Arunyingmongkol $\mathrm{K}$, Wilde H, Streptococcus suis meningitis in Thailand, Southeast Asian J Trop Med Public Health 2004; 35(4): 868-876.

13. Dejace J, Bagley P, Wood E. Streptococcus suis meningitis can require a prolonged treatment course, Int J Infect Dis 2017; 65: 34-36.

14. Mai NT, Hoa NT, Nga TV, Linh ID, Chau TT, Sinh DX, et al. Streptococcus suis meningitis in adults in Vietnam, Clin Infect Dis 46(5) (2008) 659-667. 